TITLE:

\title{
Chiroptical properties of cholesteric liquid crystals of chitosan phenylcarbamate in ionic liquids
}

\section{$\operatorname{AUTHOR}(\mathrm{S})$ :}

Sato, Junichi; Morioka, Nobuhiro; Teramoto, Yoshikuni; Nishio, Yoshiyuki

\section{CITATION:}

Sato, Junichi ... [et al]. Chiroptical properties of cholesteric liquid crystals of chitosan phenylcarbamate in ionic liquids. Polymer Journal 2014, 46(9): 559-567

\section{ISSUE DATE:}

2014-05-21

URL:

http://hdl.handle.net/2433/198803

\section{RIGHT:}

(C) 2015 The Society of Polymer Science, Japan; この論文は出版社版で ありません。引用の際には出版社版をご確認ご利用ください。; This is not the published version. Please cite only the published version. 


\section{Chiroptical Properties of}

\section{Cholesteric Liquid Crystals of}

\section{Chitosan Phenylcarbamate in Ionic Liquids}

Junichi Sato, Nobuhiro Morioka, Yoshikuni Teramoto, and Yoshiyuki Nishio*

Division of Forest and Biomaterials Science, Graduate School of Agriculture, Kyoto University, Sakyo-ku, Kyoto 606-8502, Japan

ABSTRACT: Chitosan phenylcarbamate (CtsPC) samples were synthesized to have different degrees of substitution (DS) ranging from 2.3 to 3.5, and their lyotropic liquid crystallinity in ionic liquids was investigated. 1-Ethyl-3-methylimidazolium dicyanamide ([C2Mim] $\left.\left[\mathrm{N}(\mathrm{CN})_{2}\right]\right)$ was found to be a novel solvent for CtsPC, and the concentrated lyotropic system formed a cholesteric liquid-crystalline phase and mostly exhibited vivid colorations due to selective reflection of visible light. Ultraviolet-visible-near-infrared spectroscopy and wide-angle X-ray diffractometry were conducted for evaluating the maximum wavelength of the selective light reflection, and the distance and azimuth difference between adjacent nematic thin layers in the cholesteric mesophase, respectively. The cholesteric helical pitch increased with decreasing polymer concentration, with increasing temperature, or with increasing DS. The helical sense remained right-handed 
under the adopted measurement conditions, although CtsPC are known to form a left-handed cholesteric structure in polar organic solvents such as $N, N$-dimethylformamide (DMF). Accordingly, an inversion of the cholesteric sense was achieved by combination of [C2Mim] $\left[\mathrm{N}(\mathrm{CN})_{2}\right]$ and DMF as solvent; circular dichroism and optical rotatory dispersion spectroscopy verified that the sense of CtsPC cholesterics changed from left-handed to righthanded with increasing [C2Mim] $\left[\mathrm{N}(\mathrm{CN})_{2}\right]$ content in the mixed solvent.

Running head; Cholesteric liquid crystals of chitosan derivatives

Keywords; Chiroptical property/Chitosan/Ionic liquid/Liquid crystal/Phenylcarbamate

\section{INTRODUCTION}

A large number of cellulosics as polymer molecule and even cellulose microfibrils (nanocrystallites) are capable of forming an optically anisotropic mesophase in various solvents because of the inherent semi-rigidity and chirality in the carbohydrate backbone. ${ }^{1-3}$ The supermolecular structure in the mesophase is usually of a cholesteric (or chiral nematic) type, with possible exceptions such as a columnar type found for thermotropic cellulose trialkanoates. $^{4} \quad$ Although there remain unresolved problems concerning the correlation between their molecular structure and the liquid-crystalline phase structure, the general feature of the mesomorphic assembly of cellulosics seems to be firmly established. ${ }^{1-6}$ Regarding the lyotropic systems, for example, the optical properties are significantly affected by the solvent sort and composition. (Hydroxypropyl)cellulose liquid crystals in mere water are clearly colored, but the coloration is dramatically changed by addition of a small amount of inorganic salts or organo-ionic liquids (imidazolium salts) as the third component. ${ }^{7,8}$ To take another example, a literature describes that several ionic liquids (ILs) can be a direct 
solvent for chemically unmodified cellulose, and, especially, the use of 1-butyl-3methylimidazolium chloride leads to a viscous cellulose solution which can display optical birefringence at polymer concentrations of more than $10 \mathrm{wt} \% .^{9}$

Meanwhile, chitin and chitosan are the second most abundant polysaccharides after cellulose and widely distributed in crusts of marine crustaceans as well as in exoskeletons of many species of insects. These two polysaccharides are structurally similar to cellulose; chitin consists of 2 -acetamido-2-deoxy- $\beta$-D-glucose units through a $\beta(1 \rightarrow 4)$ linkage, and chitosan is an $N$-deacetylated derivative of chitin. In contrast to the situation of cellulose derivatives, there have been only a limited number of studies on liquid-crystalline behavior of chitin or chitosan derivatives, ${ }^{10-13}$ except for the mesophase characterization of chitin nanocrystals (microfibrils). ${ }^{14,} 15$ Taking one of those examples, we reported the molecular structure and liquid-crystalline characteristics of chitosan phenylcarbamate (CtsPC, Figure 1a) in polar organic solvents such as DMF, $N, N$-dimethylacetamide (DMAc), and dimethyl sulfoxide (DMSO); ${ }^{16}$ CtsPC was found to form a left-handed cholesteric arrangement, irrespective of variations in temperature, polymer concentration, DS, and the solvent species used.

\section{$<<$ Figure 1 $>>$}

Of late years, a few studies have been made on dissolution of chitin in ILs, where 1-butyl3-methylimidazolium chloride, ${ }^{17}$ 1-ethyl-3-methylimidazolium chloride, ${ }^{18}$ and 1-butyl-3methylimidazolium acetate ${ }^{19}$ were reported to be the good solvents. Such ILs have attracted a great deal of attention not only as green solvent media but also as electrolytes to achieve ion-conductive materials. ${ }^{20}$ For instance, anisotropic ion conductivity has been accomplished in liquid-crystalline layered assemblies consisting of a pair of $\mathrm{IL} /$ mesogenic molecule of modest molecular weight. ${ }^{21}$ As for polysaccharide derivatives, investigations into the thermotropic liquid-crystallinity and ion conductivity of a cellulose derivative dangling 
poly(ethylene oxide) side-chains have been pursued. ${ }^{22}$ Moreover, a lithium ion battery made up of a polymer gel of cellulose derivative/poly(ethylene glycol)dimethacrylate has been reported to show a practical level of ionic conductivity. ${ }^{23}$ These examples raise the possibility that ILs would serve as direct solvents for lyotropic liquid crystals of polysaccharide derivatives, and that such lyotropics should be used as novel devices responding to external stimuli (e.g. light, electric field, etc.). To the authors' knowledge, however, no work has been reported on the explicit liquid-crystalline phase behavior and related functionalities of chitin or chitosan derivatives in ILs as specific fluid media.

In the present paper, we examined a series of CtsPC samples for observation of a clear mesophase formation in imidazolium salts. In continuation of our study on the molecular modification and liquid crystallinity of chitosan, we aimed at elucidating the possible cholesteric ordering and ensuing optical properties of CtsPC lyotropics in relation to the molecular parameters such as DS as well as to the surrounding conditions including polymer concentration, temperature, and solvent composition.

\section{EXPERIMENTAL PROCEDURE}

Materials. Chitosan materials, lot HF-1 $\left(M_{\mathrm{v}}=1.06 \times 10^{5}\right)$ and HC-2W $\left(M_{\mathrm{v}}=1.46 \times 10^{5}\right)$ of Hokkaido Soda (Japan), were used after a regeneration treatment. ${ }^{16}$ Phenyl isocyanate (PhI), DMAc, 1-methylimidazole, iodoethane, lithium bis(trifluoromethanesulfonyl)imide, silver nitrate, and ethyl acetate were purchased from Wako Pure Chemical Industries, Ltd. and used as received. Ethyl bromide, 1-bromobutane, 1-allyl chloride, DMF, toluene, and methanol were purchased from Nacalai Tesque, Inc. and used without further purification. 1Chlorobutane and 1-bromohexane were purchased from Sigma-Aldrich Co. and used as supplied. Commercially available potassium hexafluorophosphate (Tokyo Chemical Industry Co., Ltd.) and sodium dicyanamide (Alfa Aesar GmbH \& Co.) were used as received. 
Measurements. An ultraviolet-visible-near-infrared (UV-Vis-NIR) spectrometer (HITACHI U-4100) equipped with a thermo-regulated cell-holder was used to determine selective light-reflection bands. Circular dichroism (CD) and optical rotatory dispersion (ORD) measurements were conducted with a Jasco J-820DH spectropolarimeter to examine the handedness of the cholesteric helical structure. CD spectra were also utilized to determine the selective light-reflection bands, in the case where the corresponding signal in UV-Vis-NIR spectra was too small to detect the maximum reflective wavelength. An Olympus polarized optical microscope (POM) (BX60F5) was utilized to verify the optical anisotropy of concentrated solutions, and also to observe a fingerprint pattern for cholesteric liquid crystals of a relatively long pitch. Refractive index measurements were carried out using an Abbé refractometer equipped with a thermo-regulated stage and a polarizer rotatable over the eyepiece.

The distance and an angular difference of rotation between adjacent thin layers in the cholesteric structure were estimated with the aid of wide-angle X-ray diffractometry (WAXD). The WAXD measurements were carried out at $25^{\circ} \mathrm{C}$ using a Rigaku Ultima IV in a reflection mode. Nickel-filtered $\mathrm{Cu} \mathrm{K \alpha}$ radiation was used at $40 \mathrm{kV}$ and $40 \mathrm{~mA}$. Fluid samples were relaxed for at least $24 \mathrm{~h}$ in a copper sample holder of $0.5 \mathrm{~mm}$ high, and subsequently the diffraction intensity profiles were collected in a range of $2 \theta=3-20^{\circ}$.

Synthesis and Characterization of Chitosan Phenylcarbamate. The procedure for synthesis and characterization of CtsPC was almost the same as that described in the preceding paper. ${ }^{16}$ In brief, PhI (7.5-15 equiv/glucosamine unit) was added to a chitosan/DMAc suspension (polymer conc., 3 wt \%) in a flask under a dry $\mathrm{N}_{2}$ atmosphere. The reactive system was continuously stirred at $25{ }^{\circ} \mathrm{C}$ in the closed vessel for a prescribed time period (72-144 h). The resulting homogeneous solution was added dropwise into a vigorously stirred, large excess amount of methanol. Each product obtained as a precipitate 
was filtered and purified with DMF and methanol. The purified CtsPC was treated by a Soxhlet extraction with methanol for 1 week to thoroughly remove DMF and dried at $40{ }^{\circ} \mathrm{C}$ in vacuo for $48 \mathrm{~h}$. Substitution parameters of the CtsPC products were evaluated by ${ }^{1} \mathrm{H}$ NMR spectroscopy.

Synthesis of Ionic Liquids. All the $N$-substituted imidazolium salts used were synthesized through $N$-alkylation/quaternization of 1-methylimidazole with different alkyl halides or allyl halide, and subsequent anion exchange if necessary, by reference to literatures. ${ }^{24-28}$ For instance, the procedure to obtain 1-ethyl-3-methylimidazolium dicyanamide ([C2Mim][N(CN)2], Figure 1b) was as follows: 1-Methylimidazole $(0.10 \mathrm{~mol})$ was stirred at room temperature in a round-bottomed flask equipped with a reflux condenser under a dry $\mathrm{N}_{2}$ atmosphere, and iodoethane $(0.15 \mathrm{~mol})$ was added at adequate time intervals. After the mixture was stirred at $70{ }^{\circ} \mathrm{C}$ for $18 \mathrm{~h}$, the upper phase containing unreacted reagents was decanted. The residual crude product was washed with hot toluene lightly and then with ethyl acetate thoroughly by the combined use of stirring and ultrasonic treatment. After decanting ethyl acetate, the remaining solvent was removed by heating the ionic liquid phase to $70{ }^{\circ} \mathrm{C}$ and stirred while on a vacuum line. The resultant 1-ethyl-3-methylimidazolium iodide $(0.10 \mathrm{~mol})$ was once dissolved in a small quantity of distilled water, and then the salt solution was mixed with silver dicyanamide $(0.11 \mathrm{~mol})$ that was previously prepared by mixing equimolar amounts of silver nitrate and sodium dicyanamide. After vigorous stirring at $40{ }^{\circ} \mathrm{C}$ over $5 \mathrm{~h}$, the objective product [C2Mim] $\left[\mathrm{N}(\mathrm{CN})_{2}\right]$ was purified by filtration and drying in vacuo at $60{ }^{\circ} \mathrm{C}$ for $48 \mathrm{~h}$. In an essentially similar manner, 1-butyl-3methylimidazolium chloride ([C4Mim][Cl]), 1-butyl-3-methylimidazolium bromide ([C4Mim][Br]), 1-hexyl-3-methylimidazolium bromide ([C6Mim][Br]), 1-allyl-3methylimidazolium chloride ([AMim][Cl]), 1-ethyl-3-methylimidazolium bis(trifluoromethanesulfonyl)imide $\quad$ ([C2Mim] $\left.\left[\mathrm{Tf}_{2} \mathrm{~N}\right]\right), \quad$ 1-butyl-3-methylimidazolium 
bis(trifluoromethanesulfonyl)imide ([C4Mim][Tf $2 \mathrm{~N}])$, and 1-butyl-3-methylimidazolium hexafluorophosphate ([C4Mim] $\left.\left[\mathrm{PF}_{6}\right]\right)$ were synthesized with the respective relevant reagents. The salt products were all identified as the respective objects of $N$-substituted methylimidazolium salts by ${ }^{1} \mathrm{H}$ NMR measurements and elemental analysis. The elemental analysis was conducted only to confirm no presence of halogen, i.e., completion of the anion exchange.

Preparation of CtsPC/IL Solutions. CtsPC solutions were prepared in a polymer concentration range of 5-45 wt \% by using the ILs described above. The prescribed amount of the solvent was added to CtsPC powder weighed in a hermetically sealable glass vial and placed at $25^{\circ} \mathrm{C}$ over a period of 3 month. In the procedure, the vial was turned upside down and centrifuged at intervals for the purpose of accelerating the dissolution of CtsPC.

A privileged series of CtsPC solutions were also prepared at $46 \mathrm{wt} \%$ by using a mixed solvent consisting of [C2Mim] $\left[\mathrm{N}(\mathrm{CN})_{2}\right]$ and DMF, according to the same technique as that described above.

\section{RESULTS AND DISCUSSION}

Substitution Parameters of CtsPC. ${ }^{1} \mathrm{H}$ NMR data were used for determination of the values of DS, MS (molar substitution), and $\mathrm{DP}_{\mathrm{s}}$ (degree of polymerization in the side chains) of CtsPC; where DS and MS denote the average number of phenylcarbamoyl substitution associated with the four protons of $\mathrm{C} 2-\mathrm{NH}_{2}, \mathrm{C} 3-\mathrm{OH}$, and $\mathrm{C} 6-\mathrm{OH}$ and that of all phenylcarbamoyl entities introduced, respectively, per glucosamine residue of chitosan, and $\mathrm{DP}_{\mathrm{s}}$ is defined as the average degree of phenylcarbamoyl polyaddition for the modified sites of the possible four. ${ }^{16}$ The structural parameters evaluated for the CtsPC samples used are summarized in Table 1, where CtsPC of DS $=x$ is encoded as CtsPC $x$. 
Solubility of CtsPC in Ionic Liquids. Table 2 shows the solubility of $\mathrm{CtsPC}_{2.84}$ as a representative CtsPC product in each IL solvent at room temperature $\left(25^{\circ} \mathrm{C}\right)$. CtsPC was soluble in $[\mathrm{C} 4 \mathrm{Mim}][\mathrm{Cl}]$, [C4Mim] $[\mathrm{Br}]$, and $[\mathrm{C} 6 \mathrm{Mim}][\mathrm{Br}]$ and provided an isotropic phase in the polymer concentration range of 5-10 wt \%. When the concentration was elevated to 15 or $20 \mathrm{wt} \%$, the three lyotropics all transformed into a gelatinous state although optical birefringence was noted in the CtsPC/[C4Mim][Br] system. In the employments of [AMim][Cl], [C2Mim] [Tf $2 \mathrm{~N}],[\mathrm{C} 4 \mathrm{Mim}]\left[\mathrm{Tf}_{2} \mathrm{~N}\right]$, and [C4Mim] $\left[\mathrm{PF}_{6}\right]$, CtsPC was insoluble in any of them even at a low concentration such as $5 \mathrm{wt} \%$. In [C2Mim] $\left[\mathrm{N}(\mathrm{CN})_{2}\right]$, surprisingly, CtsPC formed an anisotropic phase at polymer concentrations exceeding $15 \mathrm{wt} \%$, and dissolved even at a higher concentration of $45 \mathrm{wt} \%$ so that the solution exhibited a vivid cholesteric color. It was thus revealed that the solubility of CtsPC was significantly affected by anion species of ILs, rather than the side chain structure (e.g. alkyl chain length) of imidazolium cations.

\section{$<<$ Table 2 $>>$}

Helical Pitch of CtsPC/[C2Mim][N(CN)2] Cholesterics. Optical properties of cholesteric liquid crystals are mainly governed by the periodicity in the supermolecular helical structure. For the present CtsPC lyotropic system, the practically important cholesteric pitch $(P)$ was evaluated for the explicit dependence on the three variable factors; polymer concentration, temperature, and DS. The wavelength $\left(\lambda_{\mathrm{M}}\right)$ that gives a peak maximum of a reflection band can be related to the cholesteric pitch by the de Vries equation ${ }^{29}$

$$
\lambda_{\mathrm{M}}=\tilde{n} P
$$

where $\tilde{n}$ is an average refractive index of the mesophase. For all the CtsPC/[C2Mim] $\left[\mathrm{N}(\mathrm{CN})_{2}\right]$ samples explored in this paper, the index $\tilde{n}$ decreased with increasing temperature and with decreasing polymer concentration; nevertheless, the two variables scarcely affected the second place of decimals of the $\tilde{n}$ data. In addition, the values 
of $\tilde{n}$ were only a little scattered by the DS variation (2.35-3.51). For the calculation of $P$ by eq 1 , therefore, it was substantially sufficient to adopt $\tilde{n}=1.54$, just fitting a data of 1.540 obtained for a $40 \mathrm{wt} \% \mathrm{CtsPC}_{2.84} /[\mathrm{C} 2 \mathrm{Mim}]\left[\mathrm{N}(\mathrm{CN})_{2}\right]$ solution at $25^{\circ} \mathrm{C}$.

Figure 2 illustrates selective light reflection spectra obtained for the $40 \mathrm{wt} \%$ $\mathrm{CtsPC}_{2.84} /[\mathrm{C} 2 \mathrm{Mim}]\left[\mathrm{N}(\mathrm{CN})_{2}\right]$ solution at different temperatures under a usual condition of the incident light beam being normal to the planar cholesteric phase. These spectra data serve as a typical example demonstrating a systematic red shift in $\lambda_{\mathrm{M}}$ of the selective light reflection, accompanied by suppression in the peak intensity, with an elevation in temperature; at $\geq 70{ }^{\circ} \mathrm{C}$, the reflectance became too small to be detected. A similar spectral transformation upon heating was observed for all of the cholesteric solutions explored, differing in degree but not in direction of the peak shift. As for kinetics, the concentrated solutions $(0.2 \mathrm{~g}$, encapsulated in a cylinder-shaped vial) relatively slowly responded to the thermal stimulus; viz., complete shift of the coloration was observed $\sim 10$ min after temperature change. Therefore, the temperature-variable optical measurements were conducted following 30-min relaxation treatment to realize sufficient color shift.

\section{$<<$ Figure 2 $>>$}

Figure 3a shows the relation between $P$ and temperature for $\mathrm{CtsPC}_{2.72} /[\mathrm{C} 2 \mathrm{Mim}]\left[\mathrm{N}(\mathrm{CN})_{2}\right]$ and $\mathrm{CtsPC}_{2.84} /[\mathrm{C} 2 \mathrm{Mim}]\left[\mathrm{N}(\mathrm{CN})_{2}\right]$ solutions prepared at a fixed polymer concentration of 40 wt \%. We can see that the respective cholesteric pitches monotonically increase with elevating temperature in the limits possible for gaining the data point. This temperature dependence of cholesteric pitch will be interpreted later, in relation to the short-range order (interlayer distance and twist angle) of the cholesteric architecture. Herein, it should be noted that, as a general trend, $P$ of the CtsPC lyotropics of DS $=2.7-3.0$ increased with temperature at a much higher rate, compared with the situations in the other CtsPC series of DS $<2.7$ or DS $>$ 3.0. In accordance with the higher sensitivity of $P$ referring to the former class, 
visually, the $\mathrm{CtsPC}_{2.72} /[\mathrm{C} 2 \mathrm{Mim}]\left[\mathrm{N}(\mathrm{CN})_{2}\right]$ solution was greenish at $-10{ }^{\circ} \mathrm{C}$ and turned orangecolored via yellowish as the temperature was raised to $60{ }^{\circ} \mathrm{C}$, and, ultimately, it became uncolored at $70{ }^{\circ} \mathrm{C}$. The $\mathrm{CtsPC}_{2.84} /[\mathrm{C} 2 \mathrm{Mim}]\left[\mathrm{N}(\mathrm{CN})_{2}\right]$ solution was generally reddish at a temperature range of $-10-60{ }^{\circ} \mathrm{C}$, but the hue became definitely profound with increasing temperature, and, at $70{ }^{\circ} \mathrm{C}$, the sample was totally uncolored. However, the two solutions were still anisotropic right after discoloring at $70{ }^{\circ} \mathrm{C}$ and became isotropic directly at $90{ }^{\circ} \mathrm{C}$, which was confirmed by POM observation. The discoloration and isotropic transition points were both inclined to shift upward when the polymer concentration was raised. Concerning the cholesteric helical sense, as summarized later, it was always right-handed as far as the CtsPC solutions retained this type of mesophase below the individual isotropic transition temperature.

\section{$<<$ Figure 3 $>>$}

Figure $3 b$ embodies the concentration dependence of the cholesteric pitch for the $\mathrm{CtsPC}_{2.72} /[\mathrm{C} 2 \mathrm{Mim}]\left[\mathrm{N}(\mathrm{CN})_{2}\right]$ and $\mathrm{CtsPC}_{2.84} /[\mathrm{C} 2 \mathrm{Mim}]\left[\mathrm{N}(\mathrm{CN})_{2}\right]$ series at $25{ }^{\circ} \mathrm{C}$. In both series, the pitch decreased almost linearly in proportion to CtsPC concentration. The negative correlation between the pitch and concentration seems to be a natural phenomenon, taking into consideration the increase in relative density of the solute polymer in the cholesteric mesophase; however, caution should be exercised in this interpretation because the opposite case (i.e., positive correlation) is actually known, for instance, for a lyotropic system of cellulose triphenylcarbamate/diethylene glycol monoethyl ether. ${ }^{5}$ In this context, the concentration dependence of the short-range order in the CtsPC/[C2Mim] $\left[\mathrm{N}(\mathrm{CN})_{2}\right]$ cholesterics will be investigated in the next section.

In the use of the CtsPC products of DS $=2.7-3.0$, the reflective coloration of the cholesteric solutions had a stronger monochromaticity and was sensitive to the concentration change; viz., the vivid color moved from the red side to blue side in the spectral array, quite 
sharply, as the polymer concentration increased (Figure 4). This is probably because DS $\approx$ 2.8 (MS $\approx 3.0$ ) is the optimum substitution for CtsPC to form a well-ordered cholesteric mesophase in terms of the molecular structure, and simultaneously the helical assembly of such a CtsPC is highly responsive in the periodicity to a small fluctuation of surrounding conditions including temperature and solvent concentration.

\section{$<<$ Figure 4 $>>$}

Figure 5 exemplifies the DS dependence of cholesteric pitch for $40 \mathrm{wt} \%$ CtsPC/[C2Mim $]\left[\mathrm{N}(\mathrm{CN})_{2}\right]$ samples at $25^{\circ} \mathrm{C}$. As can be seen from the plot, the increase in DS from 2.35 to 3.51 leads to a marked rise in $P$, the overall increment being more than $130 \mathrm{~nm}$. The steepest increase of $P$ is observed at around DS $=2.8$, followed by saturation in the higher DS range of $\geq 3.0$. This example indicates that the supramolecular helicoid of CtsPC of $\mathrm{DS} \approx 2.8$ is also sensitive in the twisting power to a small fluctuation of DS itself. In association with this pitch-DS relation, DS dependence of the short-range ordering in the cholesterics will be discussed below.

\section{$<<$ Figure 5 $>>$}

Short-Range Order in Mesophase of CtsPC/[C2Mim][N(CN)2]. Figure 6 illustrates WAXD intensity profiles measured for $\mathrm{CtsPC}_{2.84} /[\mathrm{C} 2 \mathrm{Mim}]\left[\mathrm{N}(\mathrm{CN})_{2}\right]$ cholesterics of $36-44$ wt $\%$ at $25^{\circ} \mathrm{C}$. As can be seen there, all of the diffraction intensity curves gave virtually only one significant peak at a lower angular position of $2 \theta=5.0-5.5^{\circ}$, except for a diffuse scattering halo centering $2 \theta \approx 20^{\circ}$. Therefore, the CtsPC/[C2Mim] $\left[\mathrm{N}(\mathrm{CN})_{2}\right]$ solutions are found to assume an essentially nematic structure, and the short-range ordering of less than a few nanometers in the mesophase is analyzable in terms of a pseudohexagonal packing of the molecular chains. Then the distance $(d)$ between the nematic layers stacking in the mesophase can be directly estimated from the low-angle peak. The following values were estimated by Bragg's equation $(\lambda=2 d \sin \theta, \lambda=0.1542 \mathrm{~nm})$ for the five samples given in 
Figure $6 ; d=1.80,1.73,1.71,1.65$, and $1.61 \mathrm{~nm}$, decreasing gradually with increasing CtsPC concentration (Figure 7a). Temperature dependence of $d$ was also evaluated for the 40 wt \% CtsPC ${ }_{2.84} /[\mathrm{C} 2 \mathrm{Mim}]\left[\mathrm{N}(\mathrm{CN})_{2}\right]$ sample; the $d$ value slightly fluctuated from 1.71 to 1.72 $\mathrm{nm}$ with increasing temperature from 25 to $60^{\circ} \mathrm{C}$ (Figure $7 \mathrm{~b}$ ). Moreover, DS dependence of $d$ was examined at $25{ }^{\circ} \mathrm{C}$ for $40 \mathrm{wt} \%$ solutions of various CtsPC products in [C2Mim] $\left[\mathrm{N}(\mathrm{CN})_{2}\right]$; the distance gained a little increment from 1.66 to $1.72 \mathrm{~nm}$ with an increase in DS from 2.35 to 3.51 (Figure 7c). To sum up by comparison, $d$ depended on CtsPC concentration to an appreciable extent, but it was hardly affected by temperature and DS.

\section{$<<$ Figure 6 $>>$ \\ $<<$ Figure $7>>$}

Subsequently, the cholesteric twist angle $(\varphi)$, defined as an azimuth difference between adjacent nematic thin layers, was calculated from the relation $\varphi=360^{\circ} \mathrm{d} / \mathrm{P}$ by additional use of the cholesteric pitch data obtained in optical measurements. In the $\mathrm{CtsPC}_{2.84} /[\mathrm{C} 2 \mathrm{Mim}]\left[\mathrm{N}(\mathrm{CN})_{2}\right]$ lyotropics at $25{ }^{\circ} \mathrm{C}$ (Figure $7 \mathrm{a}$ ), the value of $\varphi$ increased almost linearly from 1.35 to $1.59^{\circ}$ with increasing CtsPC concentration (36-44 wt \%). Considering the diminution in $d$ commensurate to the concentration increment, we ultimately find that the decrease in the layer spacing and the increase in the twist angle, each accompanying the concentration increase, are both responsible to the negative concentration dependence of the cholesteric pitch. In Figure 7b, the variation of $\varphi$ with temperature is estimated for the 40 wt \% CtsPC ${ }_{2.84} /[\mathrm{C} 2 \mathrm{Mim}]\left[\mathrm{N}(\mathrm{CN})_{2}\right]$ solution; the value decreased linearly from 1.45 to $1.30^{\circ}$ with increasing temperature from 25 to $60{ }^{\circ} \mathrm{C}$. Since the layer spacing $d$ was hardly changed by the temperature elevation, it can be deduced that the correlation of $P$ with temperature was determined only by the variable twist angle. Lastly, the twist angle was evaluated for the 40 wt \% lyotropics of different CtsPCs (DS = 2.35-3.51) in [C2Mim] $\left[\mathrm{N}(\mathrm{CN})_{2}\right]\left(25^{\circ} \mathrm{C}\right)$, and the 
resulting values $\left(1.70-1.28^{\circ}\right)$ are plotted in Figure 7c. In this plot, the steepest decrease of $\varphi$ with increasing DS occurs at DS $\approx 2.8$, while the slopes in the ranges of DS $<2.6$ and of DS $>3.0$ are quite gentle. On the other, the increment in $d$ caused by the DS elevation was, substantially, negligibly small; hence, the distinctive variation of $P$ shown in Figure 5 is attributable to the variation of $\varphi$. The cholesteric interlayer interaction, possibly associated with a chiral conformation (e.g., helical or twisted form) of the constituent CtsPC chains, would change in strength sensitively responding to the DS variation in the range of 2.6-3.0. As a natural assumption, the sharp response of $P$ to surrounding conditions observed for the CtsPC products of DS $=2.7-3.0$ can also be ascribed to the definitive $\varphi-$ DS correlativity.

Handedness of Cholesteric Mesophase. CD spectroscopy is a useful tool, providing information about both of the pitch and rotational sense specifying a cholesteric helix. In the present measurements, most of the observed CD signals were so large that their peak top was often out of the measurable range, even when the sample thickness was reduced to $100 \mu \mathrm{m}$. Therefore, those spectra were used principally to determine the handedness of the cholesteric helical structure. As a result, all of the lyotropics of the CtsPC products in [C2Mim] $\left[\mathrm{N}(\mathrm{CN})_{2}\right]$ gave a "negative" pseudo-Cotton effect in the wavelength range of the selective light reflection, irrespective of variations in temperature $\left(-10-60^{\circ} \mathrm{C}\right)$, polymer concentration (30-45 wt \%), and DS (2.35-3.51). This makes an utter contrast to the "positive" effect in the use of polar aprotic solvents. ${ }^{16}$ It was thus verified that we realized a "right-handed" cholesteric arrangement for the chitin/chitosan-based derivative. However, it is difficult from the above experiments to clarify the reason why the cholesteric helical sense changes depending on solvent species, due to the lack of lucid data linking conformation of the polysaccharide molecules directly to their chiral liquid-crystalline structure. As far as the authors can infer, it may be possible that electric dipole moments of solvents affect that of the 
urethane-linked phenylcarbamoyl substituents, leading to alteration of some secondary structure of CtsPC molecules and the inverse of cholesteric sense as well.

Inversion of Cholesteric Sense by Changing Solvent Composition. In our previous work, ${ }^{16}$ CtsPC lyotropics with a polar aprotic solvent such as DMF, DMAc, or DMSO always formed a left-handed cholesteric structure. Accordingly, acting on a possibility of observing the inversion of cholesteric sense, we newly prepared solutions of three CtsPCs (DS $=2.72$, 3.02, and 3.51) at a fixed concentration of 46 wt \% by using a mixed solvent (MxS) consisting of $[\mathrm{C} 2 \mathrm{Mim}]\left[\mathrm{N}(\mathrm{CN})_{2}\right]$ and DMF. Hereinafter, a CtsPC $\mathrm{C}_{x}$ solution whose solvent composition is $[\mathrm{C} 2 \mathrm{Mim}]\left[\mathrm{N}(\mathrm{CN})_{2}\right]: \mathrm{DMF}=n:(10-n)$ in weight is encoded as $\mathrm{CtsPC}_{x} / \mathrm{MxS}_{n /(10-n)}$. Figure 8 shows visual appearance of a series of $\mathrm{CtsPC}_{3.02}$ lyotropics in $\mathrm{MxS}$ at $25^{\circ} \mathrm{C}$. Four samples of $\mathrm{CtsPC}_{3.02} / \mathrm{MxS}_{0 / 10}, \mathrm{CtsPC}_{3.02} / \mathrm{MxS}_{2 / 8}, \mathrm{CtsPC}_{3.02} / \mathrm{MxS}_{8 / 2}$, and $\mathrm{CtsPC}_{3.02} / \mathrm{MxS}_{10 / 0}$, imparted vivid reflection colors, while such coloration was never perceived for $\mathrm{CtsPC}_{3.02} / \mathrm{MxS}_{4 / 6}$ and $\mathrm{CtsPC}_{3.02} / \mathrm{MxS}_{6 / 4}$; however, any of the six was thoroughly anisotropic in POM observations.

\section{$<<$ Figure 8 $>>$}

For the $\mathrm{CtsPC}_{3.02} / \mathrm{MxS}$ lyotropics excluding $\mathrm{CtsPC}_{3.02} / \mathrm{MxS}_{6 / 4}$ only, the determination of $\lambda_{\mathrm{M}}$ was successfully made by UV-Vis-NIR spectroscopy covering a range of $\lambda=300-2500 \mathrm{~nm}$. In refractometry measurements for the lyotropic series $\left(25^{\circ} \mathrm{C}\right)$, the index $\tilde{n}$ increased by 0.01 in proportion as the weight fraction of [C2Mim] $\left[\mathrm{N}(\mathrm{CN})_{2}\right]$ in $\mathrm{MxS}$ increased by $20 \%$; i.e., we obtained $\tilde{n}=1.51, \quad 1.52, \quad---, \quad 1.56$ for $\mathrm{CtsPC}_{3.02} / \mathrm{MxS}_{0 / 10}, \quad \mathrm{CtsPC}_{3.02} / \mathrm{MxS}_{2 / 8}, \quad---$, $\mathrm{CtsPC}_{3.02} / \mathrm{MxS}_{10 / 0}$. Then, as to the five samples concerned, the value of each cholesteric pitch was readily calculable according to eq 1 .

With regard to the rest sample $\mathrm{CtsPC}_{3.02} / \mathrm{MxS}_{6 / 4}$, POM study revealed that the anisotropic phase was endowed with a well-developed fingerprint texture in the optical image, as illustrated in Figure 9a. This optical pattern is characteristic of cholesteric liquid crystals 
with a comparatively longer helical periodicity. The repeating distance $\left(S_{\mathrm{r}}\right)$ of the so-called retardation lines making up the pattern is taken as corresponding to half the cholesteric pitch. From a high-magnification POM data (Figure 9b), the average of $S_{\mathrm{r}}$ was assessed to be $\sim 12$ $\mu \mathrm{m}$, so that $P$ of the cholesteric $\mathrm{CtsPC}_{3.02} / \mathrm{MxS}_{6 / 4}$ would approximate $24 \mu \mathrm{m}$.

\section{$<<$ Figure 9 $>>$}

Figure 10a displays CD spectra obtained for the CtsPC 3.02 solutions mentioned above. It turns out that the derivative $\mathrm{CtsPC}_{3.02}$ formed a left-handed cholesteric mesophase in $\mathrm{MxS}_{0 / 10}$ and $\mathrm{MxS}_{2 / 8}$ and, contrastively, a right-handed cholesteric mesophase in $\mathrm{MxS}_{8 / 2}$ and $\mathrm{MxS}_{10 / 0}$. For the two samples $\mathrm{CtsPC}_{3.02} / \mathrm{MxS}_{4 / 6}$ and $\mathrm{CtsPC}_{3.02} / \mathrm{MxS}_{6 / 4}$, whose $\mathrm{CD}$ signals were too weak to rightly determine the cholesteric sense thereby, ORD spectra were also measured as shown in Figure 10b. de Vries formulated wavelength dependence of the optical rotation caused by a cholesteric liquid-crystalline domain, in the following fashion. ${ }^{29}$

$$
\alpha=\pi(\Delta n)^{2} P /\left[4 \lambda^{2}\left\{1-\left(\lambda / \lambda_{\mathrm{M}}\right)^{2}\right\}\right]
$$

where $\alpha$ is the optical rotation per unit length for light of wavelength $\lambda ; \lambda_{\mathrm{M}}$ is the maximum wavelength of selective light reflection; $\Delta n$ is birefringence of a nematic layer as structural constituent; and the cholesteric pitch $P$ here is taken to be a pseudo-scalar that is positive for a right-handed helix and negative for a left-handed helix. For the case of $\lambda / \lambda_{\mathrm{M}}<<1$, the above equation reduces to

$$
\alpha \approx \pi(\Delta n)^{2} P / 4 \lambda^{2}
$$

Therefore, in a wavelength region of $\lambda<<\lambda_{\mathrm{M}}$, it follows that $\alpha$ assumes positive or negative values when the cholesteric sense is right- or left-handed, respectively. ${ }^{1}$ Synthetically considering the results of both UV-Vis-NIR and ORD measurements, we can reasonably deduce that $\mathrm{CtsPC}_{3.02} / \mathrm{MxS}_{4 / 6}$ and $\mathrm{CtsPC}_{3.02} / \mathrm{MxS}_{6 / 4}$ formed the left-handed and right-handed arrangements, respectively, of cholesteric architecture. 
The other two series, $\mathrm{CtsPC}_{2.72} / \mathrm{MxS}$ and $\mathrm{CtsPC}_{3.51} / \mathrm{MxS}$ lyotropics, were also examined for the chiroptical characteristics in the same technical way as that described above. As to the CtsPC $_{3.51} / \mathrm{MxS}$ series, $P$ (as pseudo-scalar) was quantified only for three compositions of $[\mathrm{C} 2 \mathrm{Mim}]\left[\mathrm{N}(\mathrm{CN})_{2}\right]: \mathrm{DMF}=4: 6,5: 5$, and 6:4, because $\mathrm{CtsPC}_{3.51}$ powder was not completely soluble in [C2Mim] $\left[\mathrm{N}(\mathrm{CN})_{2}\right]$-rich $\mathrm{MxS}$ at the prescribed concentration of $46 \mathrm{wt} \%$, and, the use of DMF-rich MxS resulted in gelation of the mixture. However, it was fully confirmed that both the additional two series formed a left- or right-handed cholesteric structure, the handedness depending on the solvent composition in a similar manner to that observed for the $\mathrm{CtsPC}_{3.02} / \mathrm{MxS}$ series. To our knowledge, this is the first example of conversion of mesoscopic chirality realized by regulating solvent composition, as far as chitin or chitosan derivatives are concerned. However, such a compensation phenomenon of cholesteric pitch using a solvent mixture has been reported for polypeptide lyotropic liquid crystals ${ }^{30-32}$ and discussed in terms of the relative dielectric constants of the solvent and solute molecules. ${ }^{30,33}$ The result of the present work could serve as an experimental data useful for future studies including theoretical or computational analyses on the liquid-crystalline and molecular structures of polysaccharide derivatives.

In Figure $11, P^{-1}$ is plotted against the proportion of $[\mathrm{C} 2 \mathrm{Mim}]\left[\mathrm{N}(\mathrm{CN})_{2}\right]$ in the mixed solvent for the three CtsPC/MxS series. As can be seen from the construction, the value of $P^{-1}$ increased almost linearly with increasing [C2Mim] $\left[\mathrm{N}(\mathrm{CN})_{2}\right]$ content and the helical sense inverted at nearly the same fraction (nematic point) of $[\mathrm{C} 2 \mathrm{Mim}]\left[\mathrm{N}(\mathrm{CN})_{2}\right] \approx 0.55$, irrespective of the variation in DS of CtsPC. Furthermore, it should be added that the slope of the regression line for the $\mathrm{CtsPC}_{2.72}$ series was slightly steeper than that for the $\mathrm{CtsPC}_{3.02}$ series. This careful observation may be rationalized by a common finding for both the single-solvent systems of CtsPC cholesterics; the absolute value of $|P|$ increased with increasing DS, whichever solvent of [C2Mim] $\left[\mathrm{N}(\mathrm{CN})_{2}\right]$ and DMF was employed. Then, in the $P^{-1}$ versus 
[C2Mim] $\left[\mathrm{N}(\mathrm{CN})_{2}\right]$ faction plots for the mixed-solvent system, the data position would shift upward at $[\mathrm{C} 2 \mathrm{Mim}]\left[\mathrm{N}(\mathrm{CN})_{2}\right]=0$ and downward at $[\mathrm{C} 2 \mathrm{Mim}]\left[\mathrm{N}(\mathrm{CN})_{2}\right]=1$, with the increase in DS of the CtsPC sample used. This is responsible not only directly for the difference in inclination between the regression lines, but secondarily for the less shift of the nematic point against the varying DS value.

\section{$<<$ Figure 11 $>>$}

\section{CONCLUSIONS}

An ionic liquid (IL), [C2Mim][N(CN) 2$]$, was found to be a novel solvent for highly substituted CtsPC (DS = 2.35-3.51), and the lyotropic system formed a cholesteric mesophase at polymer concentrations exceeding $30 \mathrm{wt} \%$. The mesophase showed a negative concentration dependence, positive temperature dependence, and positive DS dependence of the supramolecular helical pitch, and accordingly imparted a variety of colorations because of the selective reflection of visible light. These variations in the cholesteric periodicity with plural dependencies, owing to the responsiveness of the twist angle (major factor) and the distance (rather minor factor) between adjacent nematic layers, were sufficiently characterized by UV-Vis-NIR and CD spectroscopy and X-ray diffractometry. Worthy of special remark is the finding that the CtsPC cholesterics of DS $\approx 2.8$ (MS $\approx 3.0$ ) showed a high sensitivity of the pitch, which was sharply changeable responding to any of the variations in polymer concentration, temperature, and DS itself.

Within the limits of the present exploration, the CtsPC/[C2Mim] $\left.\mathrm{N}(\mathrm{CN})_{2}\right]$ mesophase retained a right-handed helical structure, although CtsPC had been known to provide a lefthanded cholesteric phase in polar aprotic solvents of DMF, DMAc, and DMSO. As a result of our natural attempt, an inversion of the cholesteric sense was achieved by the combined use of [C2Mim] $\left[\mathrm{N}(\mathrm{CN})_{2}\right]$ and DMF. That is to say, regardless the DS value (ca. 2.7-3.5) of 
the tested CtsPC, an increase of the IL content in the mixed solvent was attended by the handedness conversion from left to right in the chiral supramolecular arrangement, together with a prediction of the nematic state of $P^{-1}=0$ at an intermediate proportion such as $[\mathrm{C} 2 \mathrm{Mim}]\left[\mathrm{N}(\mathrm{CN})_{2}\right]: \mathrm{DMF} \approx 0.55: 0.45$

The CtsPC lyotropic system containing an IL component may be of significance from a viewpoint of practical applications, because ILs could respond diversely to external electric perturbations. For example, the present system, as such or with supplementary modification, is potentially functional as a display material under an adequate field of electric force; a disproportionate cation/anion dislocation may induce a dynamic change of the cholesteric coloration. Moreover, a unique anisotropic ion-conduction could be realized by using the chirality-adjustable layered structure in the mixed-solvent system of CtsPC cholesterics.

\section{AUTHOR INFORMATION}

\section{Corresponding Author}

*Phone: +81 75753 6250. Fax: +81 75753 6300. E-mail: ynishio@kais.kyoto-u.ac.jp.

Supplementary information is available at Polymer Journal's website (http://www.nature.com/pj)

\section{REFERENCES}

(1) Guo, J. -X. \& Gray, D. G. in Cellulosic Polymers, Blends and Composites (ed. Gilbert R. D.) Ch. 2. (Hanser, New York, 1994). 
(2) Gray, D. G. \& Harkness, B. R. in Liquid Crystalline and Mesomorphic Polymers (eds. Shibaev, V. P. \& Lam, L.) 298-323 (Springer, New York, 1994).

(3) Revol, J. -F., Bradford, H., Giasson, J., Marchessault, R. H. \& Gray, D. G. Helicoidal self-ordering of cellulose microfibrils in aqueous suspension. Int. J. Biol. Macromol. 14, 170-172 (1992).

(4) Fukuda, T., Takada, A. \& Miyamoto, T. in Cellulosic Polymers, Blends and Composites (ed. Gilbert R. D.) Ch. 3. (Hanser, New York, 1994).

(5) Zugenmaier, P. in Cellulosic Polymers, Blends and Composites (ed. Gilbert R. D.) Ch. 4. (Hanser, New York, 1994).

(6) Nishio, Y. Material functionalization of cellulose and related polysaccharides via diverse microcompositions. Adv. Polym. Sci. 205, 97-151 (2006).

(7) Nishio, Y., Chiba, R., Miyashita, Y., Oshima, K., Miyajima, T., Kimura, N. \& Suzuki, H. Salt addition effects on mesophase structure and optical properties of aqueous hydroxypropyl cellulose solutions. Polym. J. 34, 149-157 (2002).

(8) Chiba, R., Ito, M. \& Nishio, Y. Addition effects of imidazolium salts on mesophase structure and optical properties of concentrated hydroxypropyl cellulose aqueous solutions. Polym. J. 42, 232-241 (2010).

(9) Swatloski, R. P., Spear, S. K., Holbrey, J. D. \& Rogers, R. D. Dissolution of cellose with ionic liquids. J. Am. Chem. Soc. 124, 4974-4975 (2002).

(10) Rout, D. K., Barman, S. P., Pulapura, S. K. \& Gross, R. A. Cholesteric mesophases formed by the modified biological macromolecule 3,6-O-(butyl carbamate)- $N$-phthaloyl chitosan. Macromolecules 27, 2945-2950 (1994). 
(11) Asahina, D., Miyashita, Y. \& Nishio, Y. Sen'i Gakkaishi, Synthesis of hydroxypropyl derivatives of chitosan and observation of phase behavior of their aqueous solutions. Sen'I Gakkaishi 55, 28-33 (1999).

(12) Asahina, D., Matsubara, T., Miyashita, Y. \& Nishio, Y. Synthesis of Hydroxypropyl derivatives of chitin and chitosan and observation of phase behavior of their aqueous solutions. Sen'I Gakkaishi 56, 435-442 (2000).

(13) Dong, Y., Yuan, Q., Wu, Y. \& Wang, M. Studies on the effect of substitution degree on the liquid crystalline behavior of cyanoethyl chitosan. J. Appl. Polym. Sci. 76, 2057-2061 (2000).

(14) Revol, J.-F. \& Marchessault, R. H. In vitro chiral nematic ordering of chitin crystallites. Int. J. Biol. Macromol. 15, 329-335 (1993).

(15) Gray, D. G. Chiral nematic ordering of polysaccharides. Carbohydr. Polym. 25, 277284 (1994).

(16) Kuse, Y., Asahina, D. \& Nishio, Y. Molecular structure and liquid-crystalline characteristics of chitosan phenylcarbamate. Biomacromolecules 10, 166-173 (2009).

(17) Xie, H., Zhang, S. \& Li, S. Chitin and chitosan dissolved in ionic liquids as reversible sorbents of $\mathrm{CO}_{2}$. Green Chem. 8, 630-633 (2006).

(18) Mantz, R. A., Fox, D. M., Green, J. M., III, Fylstra, P. A., De Long, H. C. \& Trulove P. C. Z. Dissolution of biopolymers using ionic liquids. Naturforsch. A 62, 275-280 (2007).

(19) Wu, Y., Sasaki, T., Irie, S. \& Sakurai, K. A novel biomass-ionic liquid platform for the utilization of native chitin. Polymer 49, 2321-2327 (2008). 
(20) Noda, A., Susan, M. A. B. H., Kudo, K., Mitsushima, S., Hayamizu, K. \& Watanabe, M. Brønsted acid-base ionic liquids as proton-conducting nonaqueous electrolytes. J. Phys. Chem. B 107, 4024-4033 (2003).

(21) Yoshio, M., Mukai, T., Kanie, K., Yoshizawa, M., Ohno, H. \& Kato, T. Layered ionic liquids: anisotropic ion conduction in new self-organized liquid-crystalline materials. Adv. Mater. 14, 351-354 (2002).

(22) Yue, Z. \& Cowie, J. M. G. Synthesis and characterization of ion conducting cellulose esters with PEO side chains. Polymer, 43, 4453-4460 (2002).

(23) Sato, T., Banno, K., Maruo, T. \& Nozu, R. New design for a safe lithium-ion gel polymer battery. J. Power Sources, 152, 264-271 (2005).

(24) Bonhôte, P., Dias, A.-P., Papageorgiou, N., Kalyanasundaram, K. \& Grätzel, M. Hydrophobic, highly conductive ambient-temperature molten salts. Inorg. Chem. 35, 11681178 (1996).

(25) MacFarlane, D. R., Forsyth, S. A., Golding, J. \& Deacon, G. B. Ionic liquids based on imidazolium, ammonium and pyrrolidinium salts of the dicyanamide anion. Green Chem. 4, 444-448 (2002).

(26) Huddleston, J. G., Visser, A. E., Reichert, W. M., Willauer, H. D., Broker, G. A. \& Rogers, R. D. Characterization and comparison of hydrophilic and hydrophobic room temperature ionic liquids incorporating the imidazolium cation. Green Chem. 3, 156-164 (2001).

(27) Wu, J., Zhang, J., Zhang, H., He, J., Ren, Q. \& Guo, M. Homogeneous acetylation of cellulose in a new ionic liquid. Biomacromolecules 5, 266-268 (2004). 
(28) Branco, L. C., Rosa, J. N., Ramos, J. J. M. \& Afonso, C. A. M. Preparation and characterization of new room temperature ionic liquids. Chem. Eur. J. 8, 3671-3677 (2002).

(29) de Vries, H. Rotatory power and other optical properties of certain liquid crystals. Acta Crystallogr. 4, 219-226 (1951).

(30) Uematsu, I. \& Uematsu, Y. Polypeptide liquid crystals. Adv. Polym. Sci. 59, 37-73 (1984).

(31) Robinson, C. Liquid-crystalline structures in polypeptide solutions. Tetrahedron 13, 219-234 (1961).

(32) Duke, R. W., Du Pré, D. B., Hines, W. A. \& Samulski, E. T. Poly( $\gamma$-benzyl Lglutamate) helix-coil transition. Pretransition phenomena in the liquid crystal phase. J. Am. Chem. Soc. 98, 3094-3101 (1976).

(33) Samulski, T. V. \& Samulski, E. T. van der Waals-Lifshitz forces in lyotropic polypeptide liquid crystals. J. Chem. Phys. 67, 824-830 (1977). 
Table 1. Starting Material and Substitution Parameters for CtsPC Samples

\begin{tabular}{lcccc}
\hline $\begin{array}{l}\text { sample } \\
\text { code }\end{array}$ & $\begin{array}{l}\text { starting } \\
\text { material }\end{array}$ & DS & MS & DP $_{\mathrm{s}}$ \\
\hline CtsPC $2.35_{2}$ & HC-2W & 2.35 & 2.41 & 1.02 \\
CtsPC 2.47 & HC-2W & 2.47 & 2.76 & 1.12 \\
CtsPC $2.72_{2}$ & HC-2W & 2.72 & 2.99 & 1.10 \\
CtsPC $_{2.84}$ & HF-1 & 2.84 & 3.01 & 1.06 \\
CtsPC $2.94_{2}$ & HF-1 & 2.94 & 3.04 & 1.03 \\
CtsPC $_{3.02}$ & HC-2W & 3.02 & 3.17 & 1.05 \\
CtsPC $_{3.05}$ & HF-1 & 3.05 & 3.29 & 1.08 \\
CtsPC $_{3.51}$ & HF-1 & 3.51 & 3.83 & 1.09 \\
\hline
\end{tabular}


Table 2. Solubility of $\mathrm{CtsPC}_{2.84}$ in Various Ionic Liquids at $25^{\circ} \mathrm{C}$

\begin{tabular}{lccccccc}
\hline ionic & \multicolumn{8}{c}{ polymer conc. (wt \%) } \\
liquid & 5 & 10 & 15 & 20 & 30 & 45 \\
\hline$[\mathrm{C} 4 \mathrm{Mim}][\mathrm{Cl}]$ & I & I & G & - & - & - \\
{$[\mathrm{C} 4 \mathrm{Mim}][\mathrm{Br}]$} & I & I & A & G & - & - \\
{$[\mathrm{C} 6 \mathrm{Mim}][\mathrm{Br}]$} & I & I & G & - & - & - \\
{$[\mathrm{AMim}][\mathrm{Cl}]$} & $\times$ & $\times$ & - & - & - & - \\
{$[\mathrm{C} 2 \mathrm{Mim}]\left[\mathrm{Tf}{ }_{2} \mathrm{~N}\right]$} & $\times$ & $\times$ & - & - & - & - \\
{$[\mathrm{C} 4 \mathrm{Mim}]\left[\mathrm{Tf}{ }_{2} \mathrm{~N}\right]$} & $\times$ & $\times$ & - & - & - & - \\
{$[\mathrm{C} 4 \mathrm{Mim}]\left[\mathrm{PF}_{6}\right]$} & $\times$ & $\times$ & - & - & - & - \\
{$[\mathrm{C} 2 \mathrm{Mim}]\left[\mathrm{N}_{\left.(\mathrm{CN})_{2}\right]}\right.$} & I & I & A & A & $\mathrm{C}$ & $\mathrm{C}$
\end{tabular}

Symbols: C, cholesteric; A, anisotropic; I, isotropic; G, gelatinous; $\times$, insoluble; -, not prepared. 
(a)

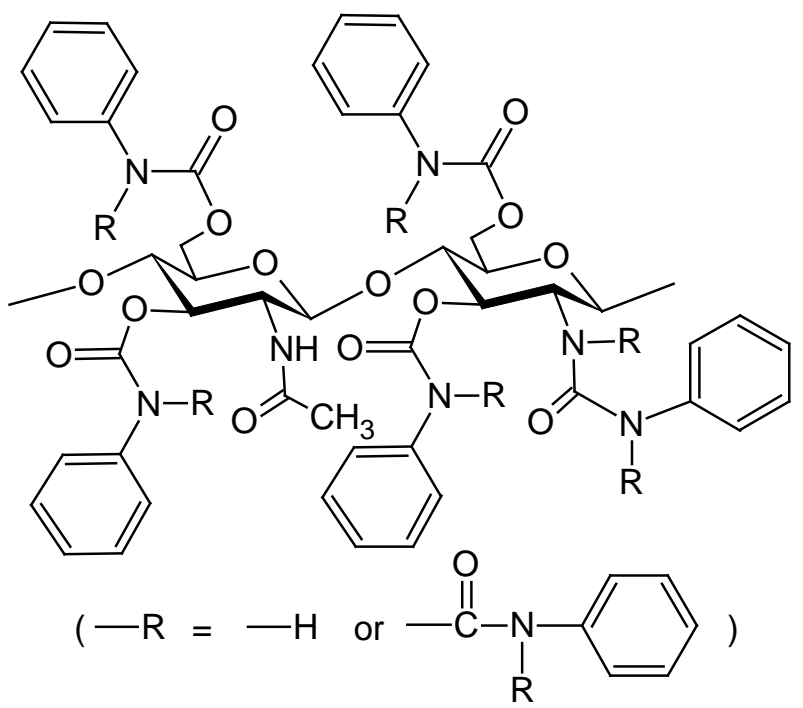

(b)

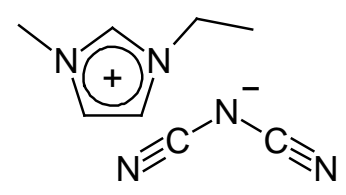

Figure 1. Structural formulae of (a) chitosan phenylcarbamate (CtsPC) and (b) 1-ethyl-3methylimidazolium dicyanamide ([C2Mim] $\left.\left[\mathrm{N}(\mathrm{CN})_{2}\right]\right)$. 


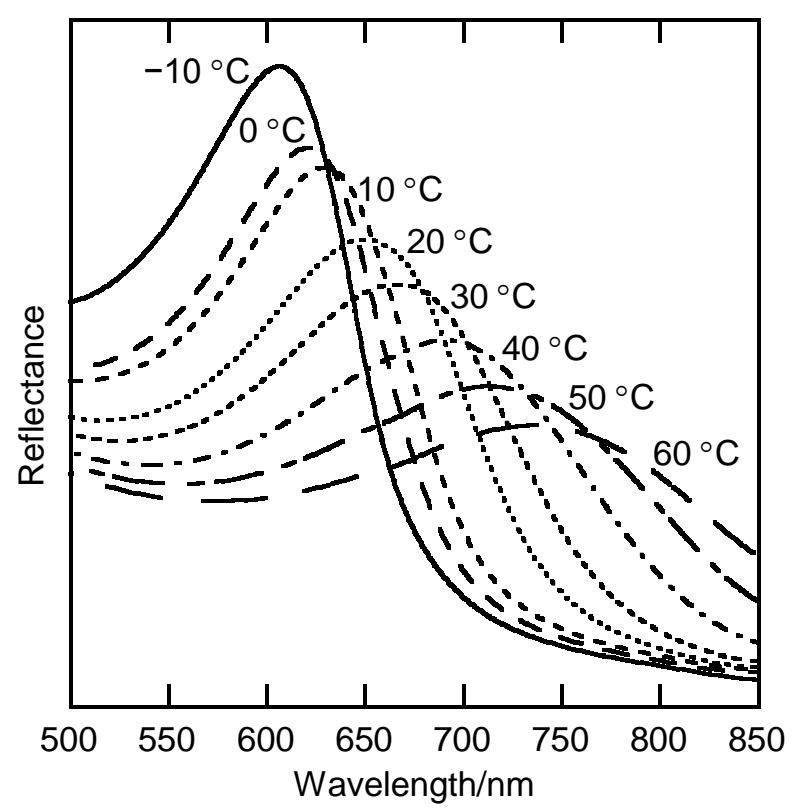

Figure 2. Selective light reflection spectra obtained for a 40 wt\% $\mathrm{CtsPC}_{2.84} /[\mathrm{C} 2 \mathrm{Mim}]\left[\mathrm{N}(\mathrm{CN})_{2}\right]$ solution at different temperatures. 


\section{(a)}
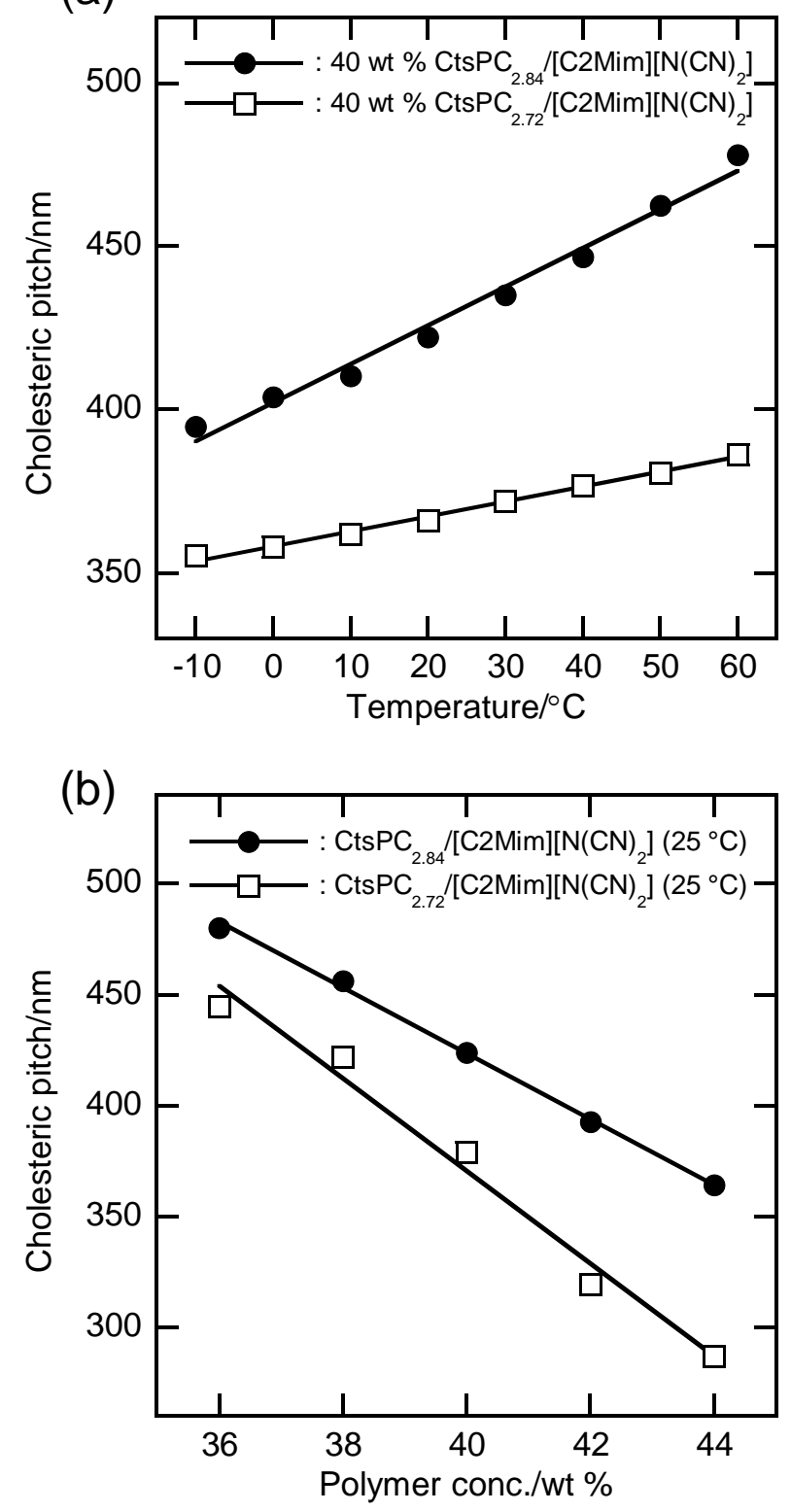

Figure 3. Cholesteric pitch for $\mathrm{CtsPC}_{2.84} /[\mathrm{C} 2 \mathrm{Mim}]\left[\mathrm{N}(\mathrm{CN})_{2}\right]$ and $\mathrm{CtsPC}_{2.72} /[\mathrm{C} 2 \mathrm{Mim}]\left[\mathrm{N}(\mathrm{CN})_{2}\right]$ series: (a) temperature dependence at a fixed polymer concentration of $40 \mathrm{wt} \%$; (b) polymer concentration dependence at a constant temperature of $25{ }^{\circ} \mathrm{C}$. 
$\begin{array}{lllll}(a) & \text { (b) } & \text { (c) } & \text { (d) } & \text { (e) }\end{array}$

(1)

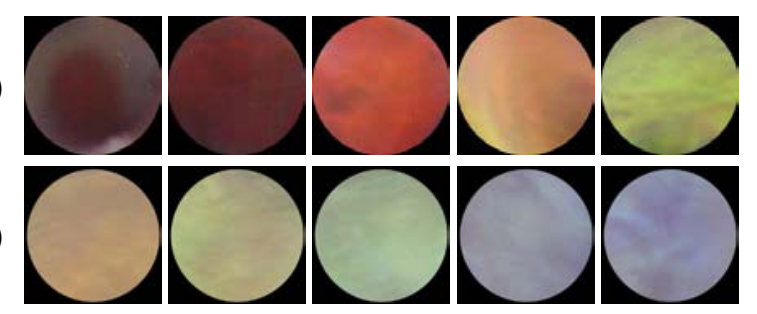

Figure 4. Photographs showing cholesteric colors for solutions of (1) $\mathrm{CtsPC}_{2.84}$ and (2) $\mathrm{CtsPC}_{2.72}$ in [C2Mim][N(CN)2]. Polymer concentration: (a) $36 \mathrm{wt} \%$, (b) $38 \mathrm{wt} \%$, (c) 40 wt \%, (d) 42 wt \%, and (e) 44 wt \%. All the photographs were obtained through digital scanning of the bottom of each individual sample vial at $25^{\circ} \mathrm{C}$. 


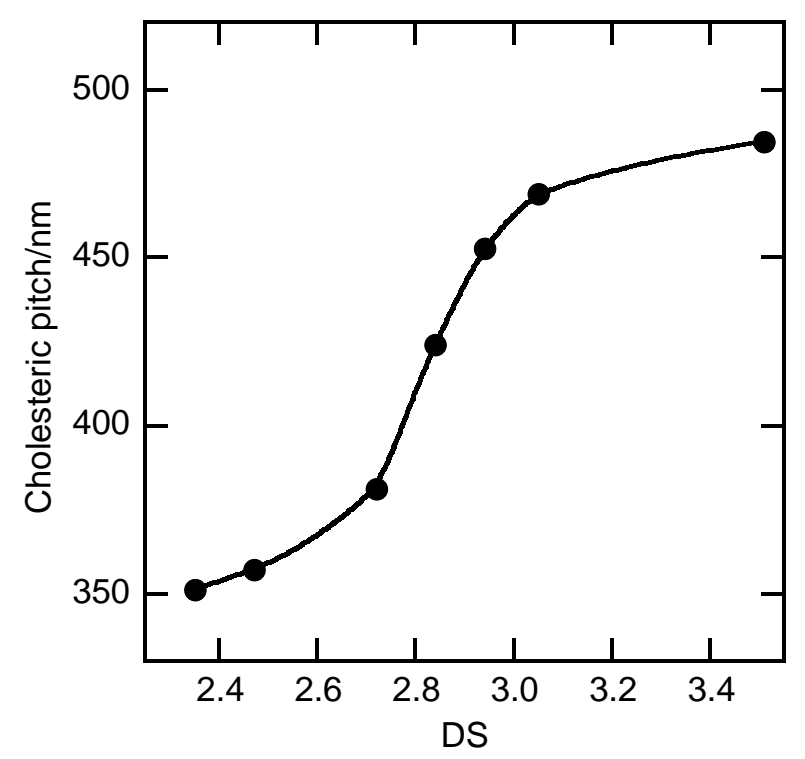

Figure 5. DS dependence of cholesteric pitch for $40 \mathrm{wt} \% \mathrm{CtsPC} /[\mathrm{C} 2 \mathrm{Mim}]\left[\mathrm{N}(\mathrm{CN})_{2}\right]$ solutions at $25{ }^{\circ} \mathrm{C}$. 


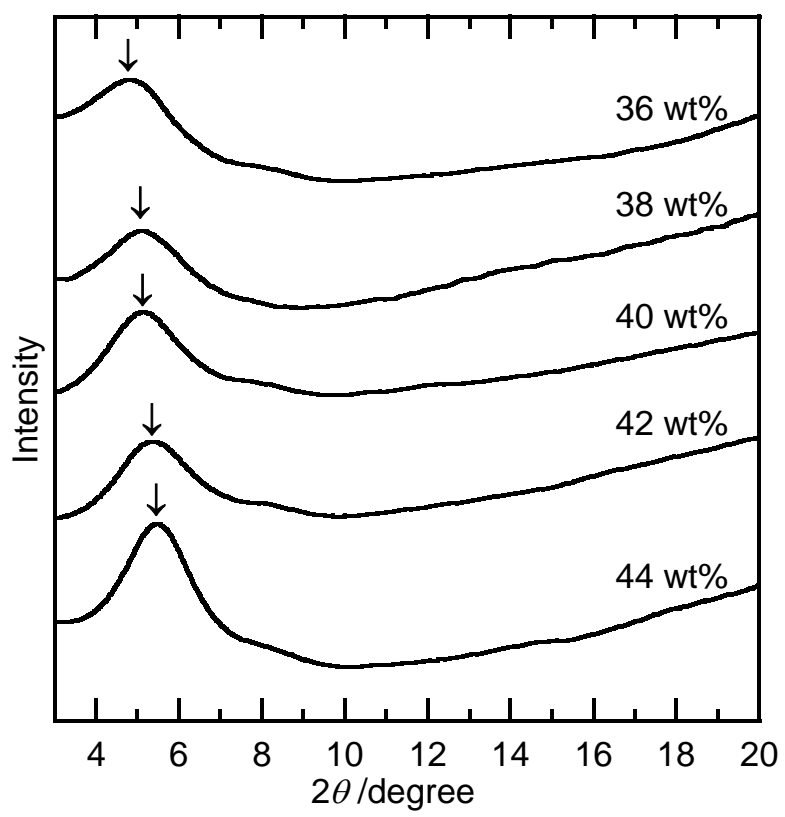

Figure 6. WAXD intensity profiles obtained for 36-44 wt \% $\mathrm{CtsPC}_{2.84} /[\mathrm{C} 2 \mathrm{Mim}]\left[\mathrm{N}(\mathrm{CN})_{2}\right]$ solutions at $25^{\circ} \mathrm{C}$. 


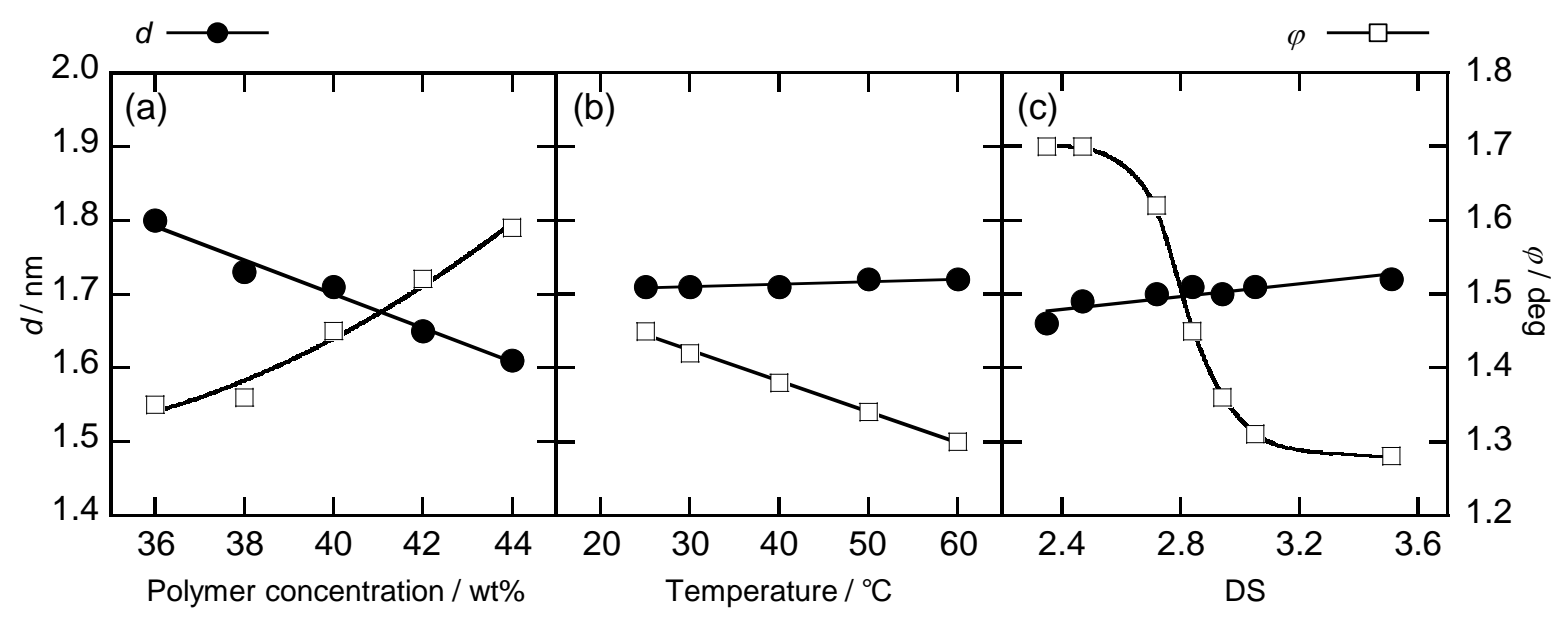

Figure 7. Plots of the interlayer distance $d$ (filled circle) and twist angle $\varphi$ (open square) for CtsPC/[C2Mim] $\left[\mathrm{N}(\mathrm{CN})_{2}\right]$ lyotropics, constructed as a function of three variables: (a) polymer concentration (at $25{ }^{\circ} \mathrm{C}$ and DS $=2.84$ ); (b) temperature (at $40 \mathrm{wt} \%$ polymer and DS = 2.84); (c) DS of CtsPC (at $25^{\circ} \mathrm{C}$ and $40 \mathrm{wt} \%$ polymer). 


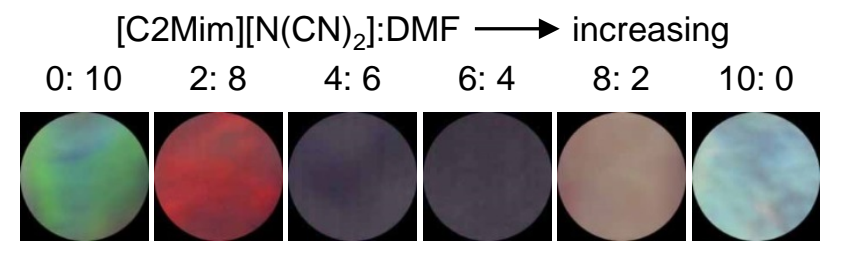

Figure 8. Photographs showing visual appearance for a series of 46 wt \% $\mathrm{CtsPC}_{3.02} /[\mathrm{C} 2 \mathrm{Mim}]\left[\mathrm{N}(\mathrm{CN})_{2}\right] / \mathrm{DMF}$ lyotropics at $25^{\circ} \mathrm{C}$. 

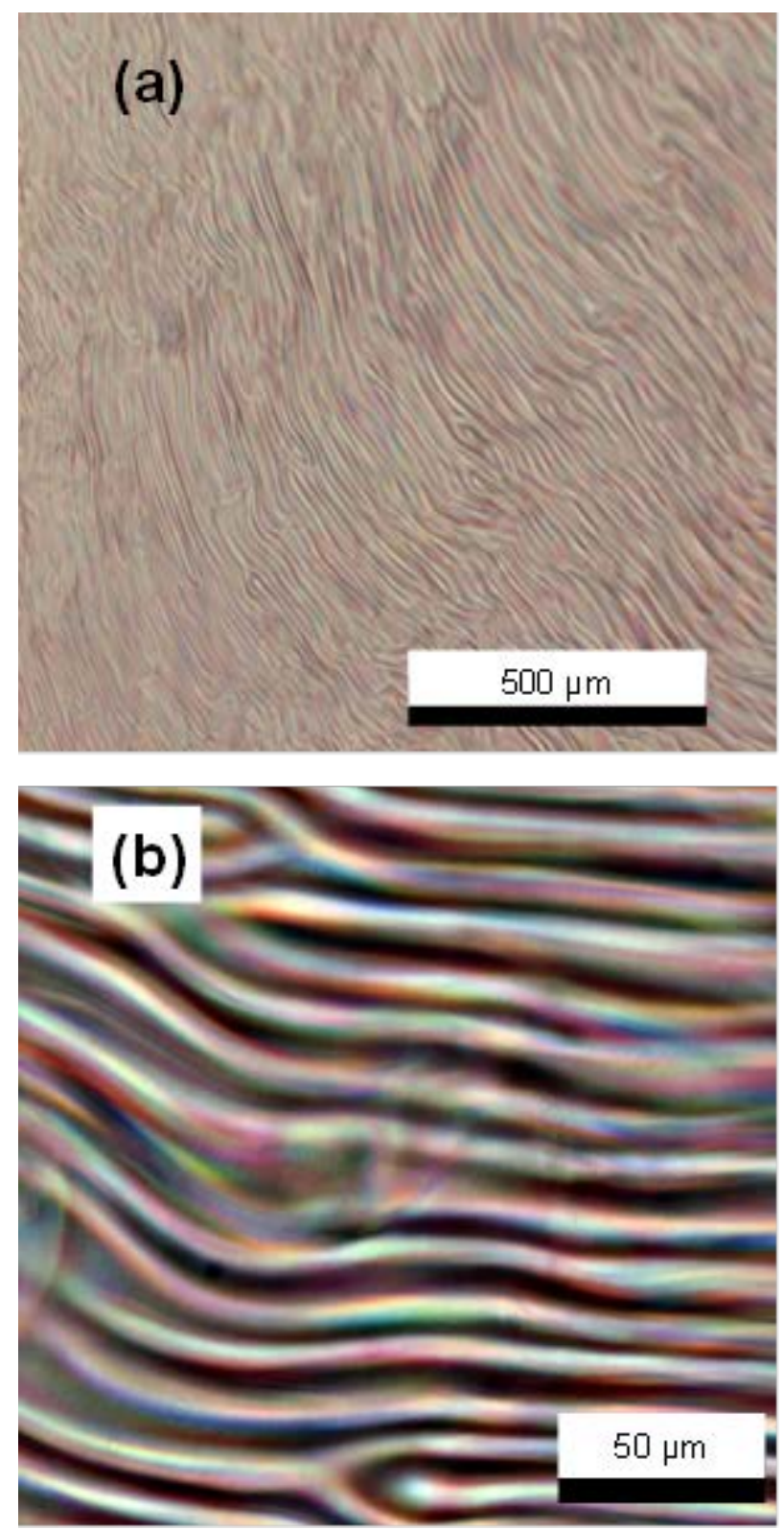

Figure 9. Polarized optical micrographs of $\mathrm{CtsPC}_{3.02} / \mathrm{MxS}_{6 / 4}$ (46 wt \% $\mathrm{CtsPC}_{3.02}$ solution in 6:4 [C2Mim] $\left.\left[\mathrm{N}(\mathrm{CN})_{2}\right] / \mathrm{DMF}\right)$. Scale bars denote $500 \mu \mathrm{m}$ in part (a), or $50 \mu \mathrm{m}$ in part (b). The sample thickness is $\sim 100 \mu \mathrm{m}$. 

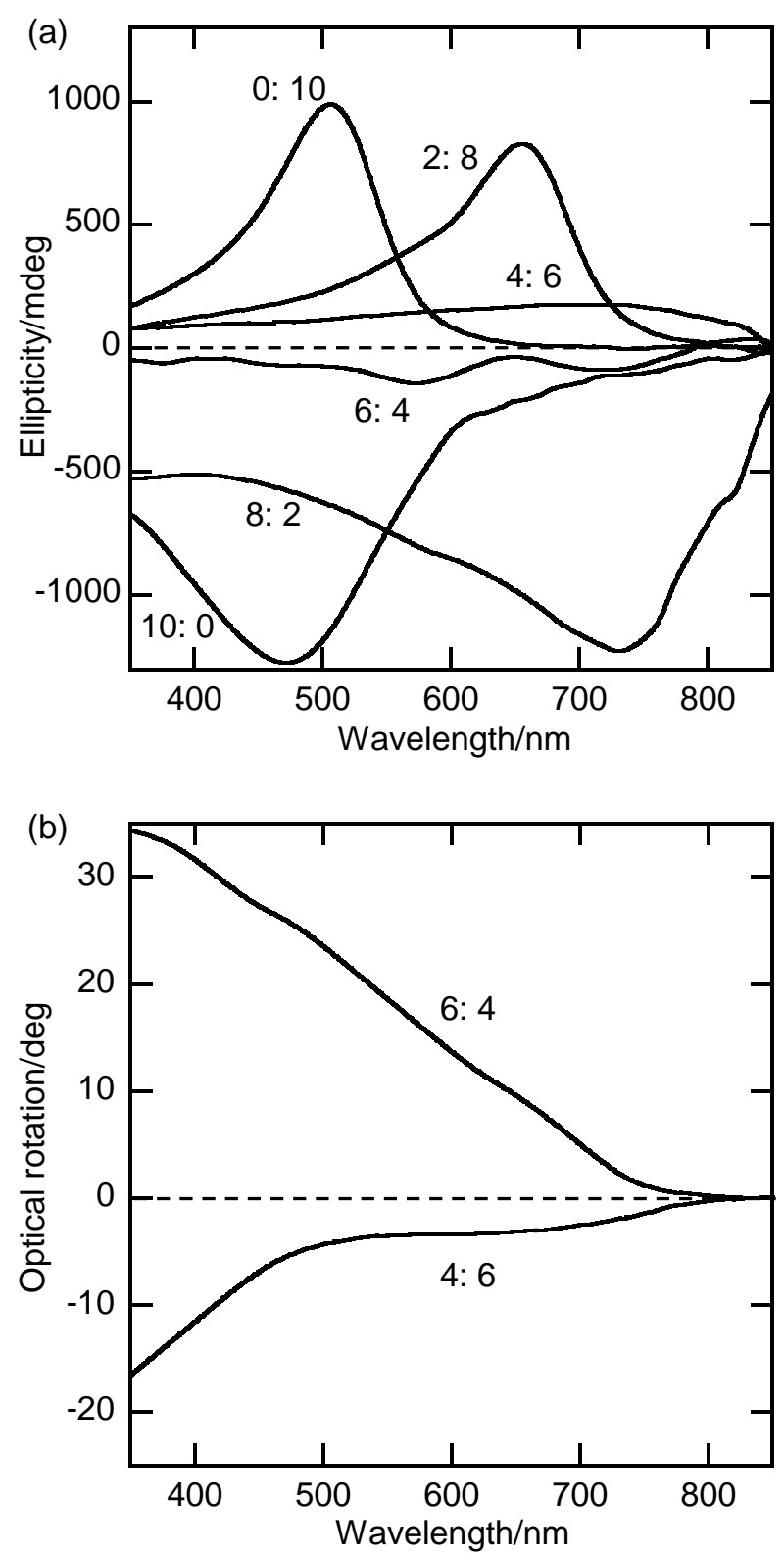

Figure 10. (a) $\mathrm{CD}$ and (b) ORD spectra obtained for the $\mathrm{CtsPC}_{3.02} /[\mathrm{C} 2 \mathrm{Mim}]\left[\mathrm{N}(\mathrm{CN})_{2}\right] / \mathrm{DMF}$ series. Numeric ratios indicate the mixed solvent composition, [C2Mim][N(CN)2]: DMF. 


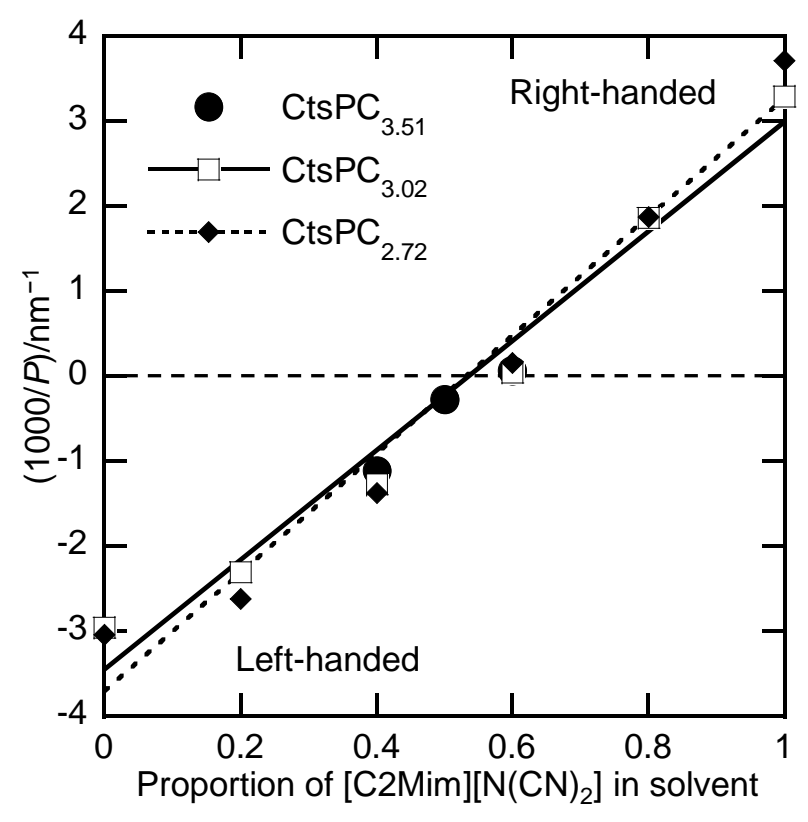

Figure 11. Plots of $1000 / P$ as a function of solvent composition for three lyotropic series of 46 wt \% CtsPC (DS = 2.72, 3.02, and 3.51) in the mixed solvent $[\mathrm{C} 2 \mathrm{Mim}]\left[\mathrm{N}(\mathrm{CN})_{2}\right] / \mathrm{DMF}$ $\left(25^{\circ} \mathrm{C}\right)$. Positive and negative signs of $P$ indicate the right-handed and left-handed cholesteric sense, respectively, and $P^{-1}=0$ corresponds to a nematic state of the mesophase. 
Table of Contents Graphic

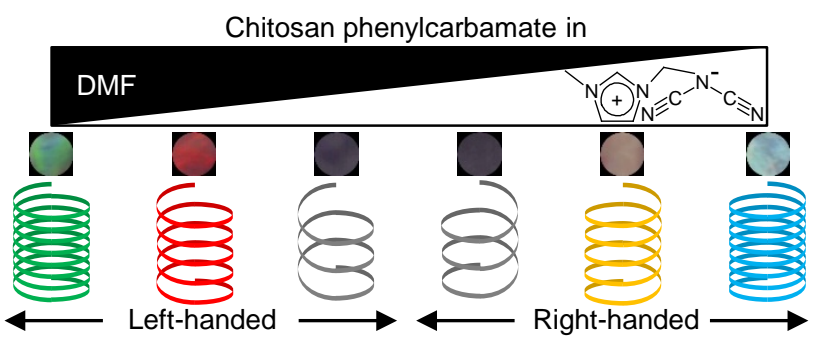

